\title{
To call a cloud 'cirrus': Sound symbolism in names for categories or items
}

\author{
Vanja Kovic $^{\text {Corresp., }}{ }^{1}$, Jelena Sucevic ${ }^{2}$, Suzy J Styles ${ }^{3}$ \\ 1 Department of Psychology, University of Belgrade, Belgrade, Serbia \\ 2 Department of Experimental Psychology, University of Oxford, Oxford, United Kingdom \\ 3 Division of Psychology, Nanyang Technological University, Singapore, Singapore \\ Corresponding Author: Vanja Kovic \\ Email address: vanja.kovic@f.bg.ac.rs
}

The aim of the present paper is to experimentally test whether sound symbolism has selective effects on labels with different ranges-of-reference within a simple nounhierarchy. In two experiments, adult participants learned the make up of two categories of unfamiliar objects ('alien life forms'), and were passively exposed to either category-labels or item-labels, in a learning-by-guessing categorization task. Following category training, participants were tested on their visual discrimination of object pairs. For different groups of participants, the labels were either congruent or incongruent with the objects. In Experiment 1, when trained on items with individual labels, participants were worse (made more errors) at detecting visual object mismatches when trained labels were incongruent. In Experiment 2, when participants were trained on items in labelled categories, participants were faster at detecting a match if the trained labels were congruent, and faster at detecting a mismatch if the trained labels were incongruent. This pattern of results suggests that sound symbolism in category labels facilitates later similarity judgments when congruent, and discrimination when incongruent, whereas for item labels incongruence generates error in judgements of visual object differences. These findings reveal that sound symbolic congruence has a different outcome at different levels of labelling within a noun hierarchy. These effects emerged in the absence of the label itself, indicating subtle but pervasive effects on visual object processing. 
To call a cloud 'cirrus': Sound symbolism in names for categories or items. Vanja Ković* ${ }^{1}$, Jelena Sučević ${ }^{2}$ and Suzy J Styles ${ }^{3}$

\author{
${ }^{1}$ Department of Psychology, University of Belgrade, Belgrade, Serbia \\ ${ }^{2}$ Department of Experimental Psychology, University of Oxford, Oxford, UK \\ ${ }^{3}$ Division of Psychology, Nanyang Technological University, Singapore
}

\section{Abstract:}

The aim of the present paper is to experimentally test whether sound symbolism has selective effects on labels with different ranges-of-reference within a simple noun-hierarchy. In two experiments, adult participants learned the make up of two categories of unfamiliar objects ('alien life forms'), and were passively exposed to either category-labels or item-labels, in a learning-by-guessing categorization task. Following category training, participants were tested on their visual discrimination of object pairs. For different groups of participants, the labels were either congruent or incongruent with the objects. In Experiment 1, when trained on items with individual labels, participants were worse (made more errors) at detecting visual object mismatches when trained labels were incongruent. In Experiment 2, when participants were trained on items in labelled categories, participants were faster at detecting a match if the trained labels were congruent, and faster at detecting a mismatch if the trained labels were incongruent. This pattern of results suggests that sound symbolism in category labels facilitates later similarity judgments when congruent, and discrimination when incongruent, whereas for item labels incongruence generates error in judgements of visual object differences. These findings reveal that sound symbolic congruence has a different outcome at different levels of labelling within a noun hierarchy. These effects emerged in the absence of the label itself, indicating subtle but pervasive effects on visual object processing.

*Corresponding author. Vanja Ković, Čika Ljubina 18-20, 11000 Belgrade, Serbia, 
29 vanja.kovic@f.bg.ac.rs 
Introduction:

When cloud watchers talk about a 'cirrus' or a 'cumulus', they are naming cloud types which can be distinguished by their visual form. The cirrus (with its voiceless sibilants, and high front vowel), refers to a high-altitude ice cloud, with a typically thin, wispy form. The cumulus, (with its sonorant consonants, and rounded vowels), refers to a puffy, low-altitude cloud, prone to rising muffin-tops. Do the sounds encoded in the names of the clouds enhance visual differences between the clouds' shapes? And if so, does an alliance of sounds with visual forms differ when labels are used at different levels of description (e.g., 'cloud' versus 'cirrus')? This nebulous example ${ }^{1}$ allows us to ask an important question about the relationship between labelling itself, and a label's 'range of reference,' for items that can be named at different levels within a noun hierarchy.

Since the first systematic studies into sound symbolism in linguistic labelling, research has tended to focus on the mapping of labels onto a small number of individual items (usually a pair), which can be discriminated on some visual dimension. For example, Sapir (1929) asked people which of the word-forms 'mil' or 'mal' would be a better label for pairs of items like a small or large table, and Köhler (1929:1947) asked whether a curved or an angular line-drawing would be a better match for word forms like 'baluma' and 'takete'. The majority of the literature on sound symbolism (for recent reviews see Imai \& Kita, 2014; Lockwood \& Dingemanse, 2015) has followed this trend for contrastive label-stimulus mappings, typically for a small number of items, usually two-dimensional line drawings, usually presented in pairs (prominent examples include Maurer, Pathman, \& Mondloch, 2006; Ramachandran \& Hubbard, 2001). Some alternative approaches include matching single words with pairs of pictures randomly generated to match certain visual characteristics of known congruence types (Drijvers, Zaadnoordijk, \& Dingemanse, 2015; Nielsen \& Rendall, 2011, 2013), or matching word-forms to a variety of natural and artificial objects, with varying agency (Flumini, Ranzini, \& Borghi, 2014). However, as the majority of methods rely on the one-to-one match between a single label

\footnotetext{
${ }^{1}$ Although this lexical pair is not a canonical example of linguistic sound symbolism, when presented with a pair of pictures showing canonically wispy cirrus (cirrus uncinus) and canonically puffy cumulus (cumulus congestus) clouds, $83 \%$ of 135 Serbian speakers unfamiliar with the formal scientific cloud names guessed which cloud is called which. For further details see the Open Science Framework repository for this article (https://osf.io/2wvug/).
} 
56

57

and single visual stimulus, it is difficult to interpret what range-of-reference these novel labels might play in naturalistic noun-labelling hierarchy - do they refer to abstract superordinate categories (e.g., animal), basic-level categories (e.g., dog), subordinate-nouns (e.g., Labrador), or even proper nouns for individual exemplars (e.g., Fido).

Research using words from unfamiliar languages provides a ready-made range-ofreference, since participants will likely bootstrap the novel word to its translation equivalent. For example, in a study by Berlin (1995), English speaking participants were above chance at guessing which label in a pair was a type of fish, and which a type of bird, for stimuli from an unfamiliar language. Studies of this kind demonstrate that sound symbolism can reside in labels for categories of natural objects, not just as one-off names for arbitrary experimental tokens: sound symbolism can enhance word learning for antonym pairs in unfamiliar languages (e.g., 'fast'/'slow') (Lockwood, Dingemanse, \& Hagoort, 2016; Nygaard, Cook, \& Namy, 2009), and facilitate word learning in tasks where adults and infants learn labels for novel action events, and extend them to new exemplars of the action (Imai, Kita, Nagumo, \& Okada, 2008; Kantartzis, Imai, \& Kita, 2011). Sound symbolism is thus not limited to arbitrary lab-based labels for abstract visual items, but is evident in natural-category labels, descriptive phrases, and action descriptions. However, among this body of research, it has remained unclear whether sound symbolism plays different roles at different levels of labelling within a single hierarchy.

There is a growing body of research suggesting that words have a special status in activating conceptual representations (Lupyan \& Thompson-Schill, 2012). For example, labels activate representations that are more abstract (Edmiston \& Lupyan, 2015), and facilitate acquisition of novel categories even when the label is redundant to the category (Lupyan, Rakinson, \& McClelland, 2007), whereas this is not the case for other correlated non-verbal cues, such as environmental sounds. The speech-specific nature of labelling on category formation has been shown in infants as young as 10-months-of-age (Althaus \& Westermann, 2016), where labels have been shown to induce visual attention to parts of objects containing featural similarities (Althaus \& Mareschal, 2014). That is to say, even for babies, hearing objects named-alike induces attention to shared object properties, and these attentional biases are thought to underly the process of categorization. Bringing categorization and sound symbolism together, a recent word learning study by Monaghan and colleagues (Monaghan, Mattock, \& 
86 Walker, 2012) showed that sound-symbolic labels facilitated learning the mapping between the

87 label and the category of items, but not between the label and the individual objects.

88

One series of studies (Kovic, Plunkett, \& Westermann, 2010) has investigated sound symbolism in labels which explicitly refer to whole categories of novel objects where multiple members of the category are available to the participant. In Kovic et al. (2010), participants learned to sort two classes of novel, schematic creatures, by allocating them to named categories. Participants began by guessing which creature belonged to which category, and with feedback, gradually refined their answers as they learned to categorize items. The creatures differed according to a number of schematic dimensions (e.g., number of legs, shape of tail), but no single feature was solely responsible for which item belonged to which category. Two groups of participants learned to form categories with labels which were sound-symbolically congruent or incongruent with the typical shape of the creatures' head (e.g., 'dom' or 'shick' for mostly-round or mostly-pointy heads). In two separate behavioural tasks, the pairings of labels to categories did not influence training speed or recall accuracy. However, during later test phases (in which people heard a label, then decided whether a subsequently presented picture matched) the congruence of the shape of the creatures' heads with the newly learned labels influenced the speed of decisions. This finding suggested that although the efficiency and success of word learning did not differ between conditions, links between the auditory label and the category members were more efficiently accessed for participants who had learned sound symbolically congruent labels.

The same study also reported electrophysiological evidence of differences between responses of the two groups of participants - during the test phase, ERPs showed characteristics of enhanced visual object processing for people who had learned the congruent category labels. Taken together, these findings suggest that when sound symbolism is present for a recently acquired category label, it enhances our ability to access information relevant to the mapping between form and meaning. In terms of mechanisms, this finding could suggest that a congruent label invokes (primes) salient features of its member items prior to their visual appearance, thus enhancing object recognition. Alternatively, encoding with symbolism may cause the individual items to be represented with richer feature representations, meaning that their individual shapes were recognized more efficiently. This could be achieved by more efficient access to the stored 
116 knowledge about each item during the test phase, following sound-symbolic encoding during 117 training.

118 However, notable questions still remain about the limits of sound symbolism in 119 naturalistic language environments. As argued in a recent review (Dingemanse, Blasi, Lupyan, 120 Christiansen, \& Monaghan, 2015), if all concepts with similar meanings had similar-sounding 121 names, the phonological space for semantically related items would become extremely crowded, 122 leading to a kind of 'systematicity' in which multiple similar form-meaning relationships 123 generate high confusability. This crowding may necessitate a tug-of-war between systematicity 124 and arbitrariness in natural language systems, with systematicity arising from similar meanings 125 being encoded by similar sounds, and arbitrariness facilitating discriminability between items 126 with similar meanings. It has therefore been suggested that systematicity plays a role in grouping 127 together categories, rather than distinguishing between exemplars. This claim has been tested on 128 meaning relations arising from different grammatical categories (Monaghan \& Christiansen, 129 2006; Monaghan, Christiansen, \& Fitneva, 2011). However, it remains to be tested whether 130 similar effects are observed for labels at different levels of noun taxonomy.

131 The aim of the present paper is to experimentally test whether sound symbolism has a 132 different effect in labels with a different range-of-reference within a simple noun-hierarchy. We 133 conducted a study in which people learned the make up of two categories of unfamiliar objects 134 ('alien life forms'), and were passively exposed to either labels for the categories, or labels for 135 each item, while they learned. Within this framework, for different groups of participants, the 136 labels were either congruent or incongruent with the objects. Since previous research (Kovic, et 137 al., 2010) has demonstrated that sound symbolism does not alter the speed or the accuracy of 138 category formation, we did not predict the influence of sound symbolism to be present during 139 training, but to emerge in tests conducted after category learning. We therefore asked whether 140 congruence between the label and the visual form during training might cause people to attend to 141 the objects differently, and influence visual object processing, even in the absence of the auditory 142 training stimulus (the label). The test phase was implemented as a silent, visual same/different 143 judgement, to tease apart whether effects are driven by sound symbolism altering the strength of 144 the mapping between the word-form and the visual features of the object, or by sound symbolism 145 altering the representation of the visual objects themselves. To the best of our knowledge, no 
146 other studies have attempted to look at differences in visual object processing caused by sound 147 symbolism, in the absence of the sound symbolic stimulus.

148 We predicted that if sound symbolism is effective in highlighting featural similarities 149 among members of a category, then congruent labels would enhance performance (faster RT and 150 fewer errors) for categories learned with category-labels, but would not influence performance 151 for categories learned with item labels. On the other hand, if sound symbolism enhances object 152 processing on a trial-by-trial basis, via a generalized form of crossmodal congruence between 153 individual sounds and individual object features, we predicted that training with congruent labels 154 would enhance performance (faster RT, fewer errors) for categories learned with all levels of 155 labelling.

\section{Method}

\section{Participants}

Eighty-two participants from a local Science Center and second-year psychology undergraduate students at the first author's institution (13 male, 17-19 years old), took part in the present experiment and received course credit for their participation. All participants reported normal or corrected-to-normal vision and were unaware of the purpose of the present study. This research was approved by one branch of the Serbian Psychological Association, the Ethics Committee from the Department of Psychology, University of Niš (document number 3/16).

Stimuli

Visual stimuli were abstract pencil drawings of novel complex shapes designed for use in a number of different studies. The forms were inspired by the shape of the historic Vinča figures, products of a Neolithic culture from the Balkan region. These abstract drawings were created to form two categories, differing in their visual properties: one category consisted of curved shapes, in rounded forms, and the other category, angular shapes, in a vertical orientation (see Figure 1B). Each category contained six members. Given their abstract forms (reminiscent of Picasso), we term these stimuli Vinčasso illustrations.

Given the novelty of stimuli, a rating task was run in order to establish that the two sets 173 of Vinčassos did indeed represent a contrast between soft/round and sharp/angular visual forms, 
174 with between category difference, and within-category homogeneity. 135 psychology and

175 education second year undergraduate students (age: 17.5-19.5 years) were asked to judge each

176 item's shape on a 7-point scale ranging from 'round' (at the leftmost side of the scale) to angular

177 (at the right). The online rating task was implemented using the Qualtrics survey platform, where

178 each item was presented in a random order. The results showed that the pencil drawn Vinčassos

179 did indeed fall into two categories (Round: $M=1.58, S D=.10$; Sharp: $M=5.68, S D=0.43$;

$180 t(5.51)=22.98, p<.001)$, with high between category difference, and high within-category

181 homogeneity. The same participants also rated the complexity of the pictures from 'simple' to

182 'complex'. The results showed no overall differences between the two Vinčasso categories

183 (Round: $M=4.3, S D=.79$; Sharp: $M=3.94, S D=.45 ; t(1,11)=.95, p=.36$ ), which showed

184 substantial within-category and within-subject variability. The original Vinčasso stimuli at 185 screen-resolution can be found in the Open Science Framework repository for this article

186 (https://osf.io/2wvug/), and further details of the rating study can be found in the Supplementary 187 Materials (S1).

188 Twelve auditory pseudowords were used as labels for the pictures. The pseduowords, 189 have a C-V-C-V-C structure, and were read aloud by the first author, a female native speaker of 190 Serbian, using a neutral intonation. In the current study, we elected to use natural speech in 191 preference to artificially generated speech, as we are interested in the naturalistic processing of 192 realistic whole language, without the potential confounds introduced by participants' awareness 193 of the unnatural characteristics of synthesized or flat-prosody speech. Stimuli were recorded in a 194 single session and filtered to remove hiss and hum using GoldWave. Stimuli were approximately 195 equal in duration $(\mathrm{M}=790 \mathrm{~ms}, \mathrm{SD}=82.3 \mathrm{~ms})$. Half of the stimuli contained soft phonological 196 structure (e.g., 'volab') and the other half, sharp phonological structure (e.g., 'šičak', See Figure 1971 for further details). The 'sharpness' and 'softness' of the phonemes were based on findings of 198 several previous studies of Serbian, the language of test, and the twelve test items were 199 previously validated for their 'sharp' and 'soft' associations (Sučević, Savić, Popović, Styles, \& 200 Ković, 2015). The audio files used for presentation in the experiment can be found in the Open 201 Science Framework repository for this article (https://osf.io/2wvug/). As it has been suggested 202 both phonetic and prosodic characteristics combine to generate sound symbolic effects 203 (Dingemanse, Schuerman, Reinisch, Tufvesson, \& Mitterer, 2016; Nygaard, et al., 2009), further 204 details about the acoustic envelope of the stimuli can be seen in the Supplementary Materials 
205 (S2), where the stimuli for the different shape conditions show substantial overlap in their 206 prosodic contours.

207

\section{Experimental design and procedure}

Participants were asked to imagine they have just landed on an unexplored planet and

they need to learn to differentiate between two species that live on the planet in order to survive. Participants were seated in front of a computer and were given headphones. Instructions were presented on screen.

The experiment consisted of a training and a test phase. In the training phase, participants

214 were familiarized with the categorical structure of the presented objects. In the first half of the training phase, members of the two categories were presented one-by-one, with an auditory label: Each trial began with a fixation cross presented for $500 \mathrm{~ms}( \pm 100 \mathrm{~ms})$, followed by the auditory label, then a single Vinčasso, approx $12 \mathrm{~cm}$ in height. While the creature remained onscreen, participants were asked to guess which of the two categories it belonged to, by pushing one of two buttons (' $\mathrm{C}$ ' and ' $\mathrm{N}$ ' on a standard keyboard), with key allocation counterbalanced across participants using $\mathrm{ABAB}$ sequence. When participants made their selection, feedback was displayed on the screen for 600ms ('Correct!' or 'Incorrect!') (Figure 1). All twelve stimuli were presented once in a random order.

In the second stage of training, labels were omitted, so that the creature was presented immediately after the fixation cross (which remained on the screen for $500 \mathrm{~ms} \pm 100 \mathrm{~ms}$ ). All twelve stimuli were presented once in a random order.

After the training phase, participants were instructed to identify whether the two creatures presented side-by-side were the same ('identical') or different. In the Test block, a fixation cross was presented for $500 \mathrm{~ms}( \pm 100 \mathrm{~ms})$, followed by the presentation of two Vinčassos side-by-side. The Creatures remained on the screen until participants responded. The test pairs depicted the same item (Identical Match), or items drawn from different categories (Category Mismatch). For mismatching pairs, each image was randomly paired with a picture from the other category, and

232 pairs were yoked across participants. 12 trials in each condition were presented in a random 
233 order, making a total of 24 trials of these types ${ }^{2}$.

\section{Training Conditions}

236 Label Type was manipulated between experiments: In Experiment 1, participants heard each

237 Vinčasso named with a different label (Item Labels), meaning that the auditory labels were not

238 meaningfully correlated with the to-be-learned category structure. In Experiment 2, participants

239 heard only two labels, each of which labeled one of the categories (Category Labels), meaning

240 that the auditory labels were correlated with the to-be-learned categories, but were redundant, as

241 the task could be solved without them.

242 Label Congruence was systematically manipulated between participants: Half of the participants

243 heard labels that were congruent with the visual properties of the Vinčassos (soft phonology 244 rounded shapes; sharp phonology - angular shapes), and half heard incongruent labels.

245 Participants were randomly assigned to a Congruence condition.

Since the categories were defined by their visual properties in both experiments (rounded, angular), the labels were an irrelevant feature of the training environment. In other words, participants could have successfully solved the category structure in this task without paying any attention to the labels. Here, we ask the question whether labels presented during a first encounter with a visual object alter subsequent visual processing of the object.

\footnotetext{
${ }^{2}$ We originally included a third condition in the Test Phase, in which participants saw different pictures from the same category. However, around two thirds of participants misunderstood the task instructions, and answered on the basis of whether the picture was from the 'same category', instead of whether the pictures were 'identical'. The large scale of the dropout rendered this condition un-analysable here (Further details can be found in the Supplementary materials S3 and the Open Science Framework repository for this project: https://osf.io/2wvug/)
} 
A.

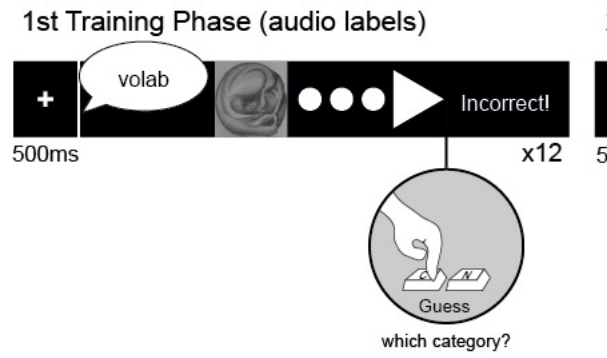

2nd Training Phase (silent) Test

B.

Training Sets Item Labels
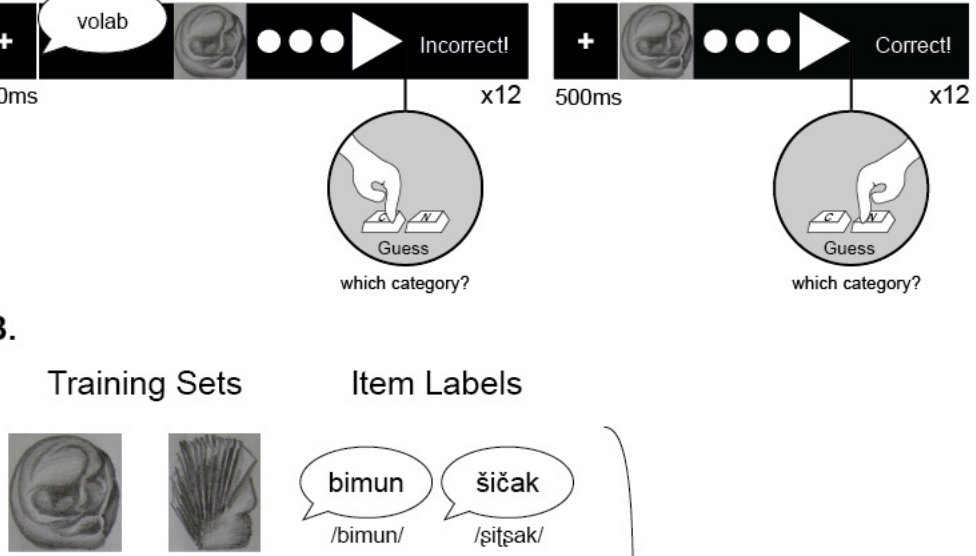

$500 \mathrm{~ms}$
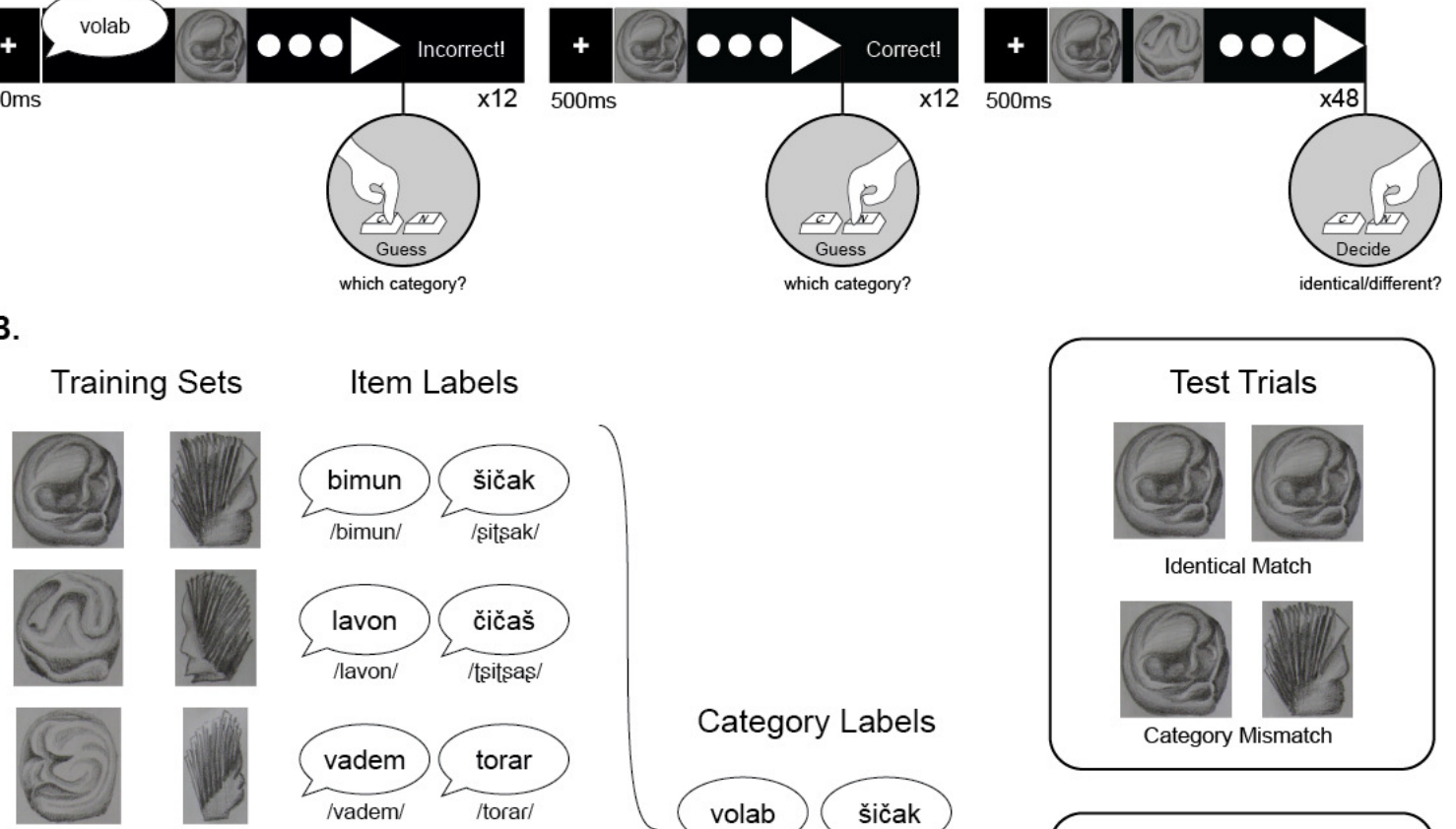

Category Labels
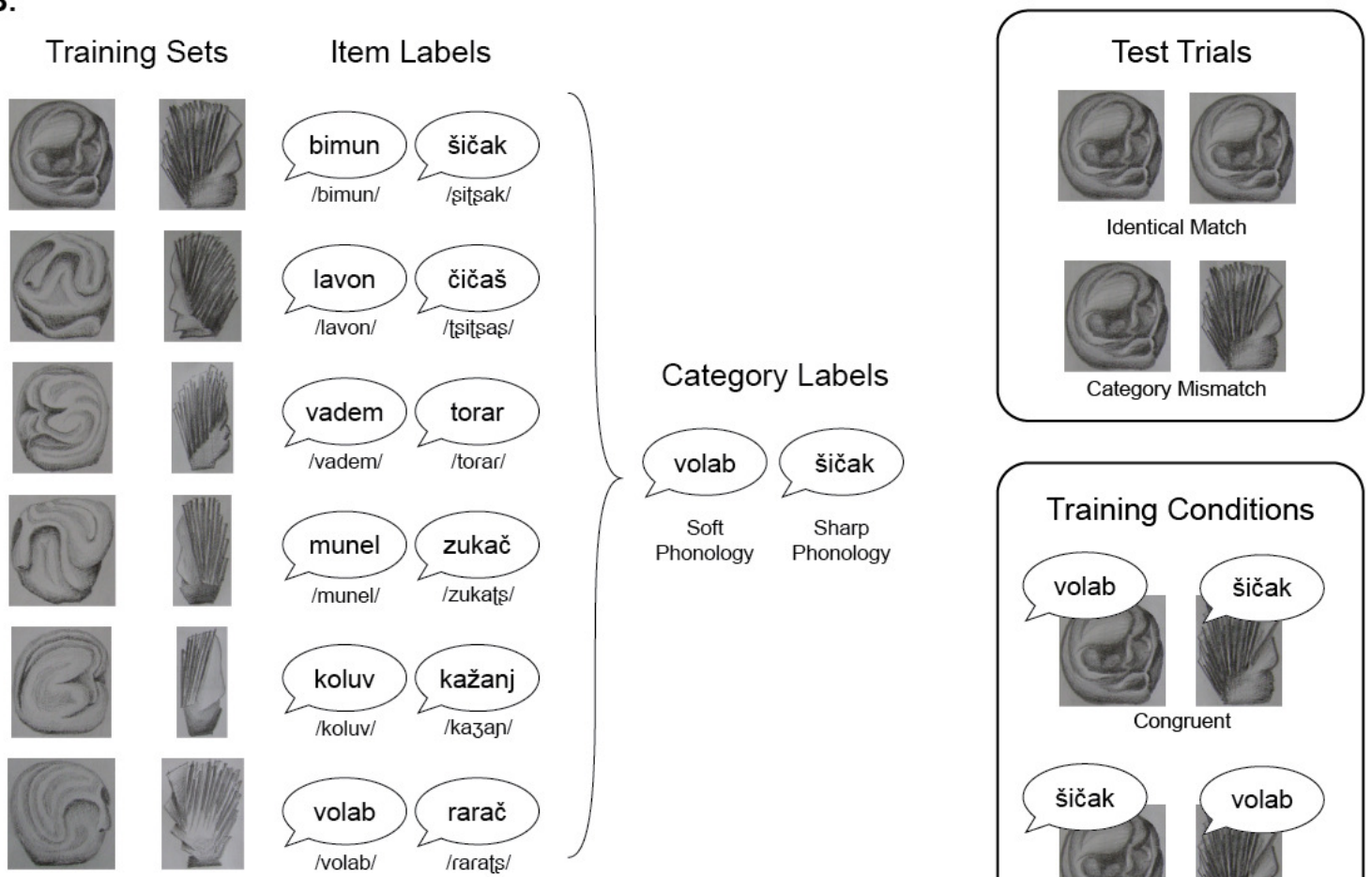

Curved
Vincassos

Angular Vincassos

$\begin{array}{cc}\text { Soft } & \text { Sharp } \\ \text { Phonology } & \text { Phonology }\end{array}$

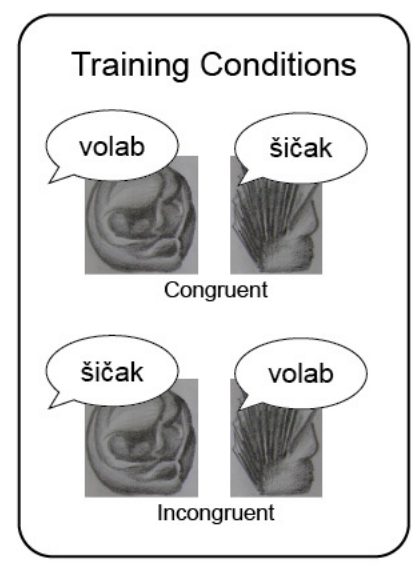

Figure 1. Experimental Design. A. The three blocks of the study, showing the structure of trials, and event timing. B. Stimuli and experimental conditions. Structure of the visual categories, and the twelve auditory labels used in Item Label training condition (names are given in standard Serbian Latin orthography, and glossed in IPA), and the 2 labels used in the Category Label training condition, along with examples of the test trial types, and the training conditions.

Mean error rates were computed for individuals in each of the four training conditions,

262 and each of the three types of test trial. Reaction times were transformed into inverse RT 263 (iRT=1/RT), to approximate normal distribution for each participant. Based on visual inspection 
264 of the distribution, extreme outliers (exceeding 100-4000ms) were excluded from further 265 analysis. For correct trials each individual's mean inverse RT was computed. Following analysis, 266 reaction times were back-transformed into milliseconds ( $\mathrm{RT}=1 / \mathrm{iRT})$ for graphing, and for 267 presentation of means.

For analysis of the training phase, mixed ANOVAs investigated the influence of Training Condition (Congruent, Incongruent) on Error Rate and on Reaction Time across the two training Blocks (First, Second). For analysis of the test trials, mixed ANOVAs investigated the influence of Training Condition (Congruent, Incongruent) on Error Rate and Reaction Time across the two match types (Identity Match, Mismatch). Power analyses were conducted using G*Power 3.1 (Faul, Erdfelder, Buchner, \& Lang, 2009).

\section{Experiment 1: Item Labels}

276

277

278

279

280

281

282

283

284

285

Each Vinčasso was presented with a different label during the first training block. Half of the participants heard congruent, and half, incongruent item labels. Labels were yoked to pictures for all participants in a given condition. The categorization task involved two categories of six visually coherent items (See Figure 1).

\section{Results and Discussion}

Training. Figure 2 shows the results from the training and the test phase of Experiment 1, with the means for individual participants shown separately. Means are given in Table 1. Participants solved the categorization task, with a reduction in errors from the first to the second block (Error: $F(1,40)=31.55, p<.001,{ }_{\mathrm{p}} \eta^{2}=.44$, Observed Power $\left.=1.00\right)$. No further effects were observed for Error Rate or RT. As seen elsewhere (Kovic, et al., 2010) in this training-by-guessing paradigm, sound symbolism is not observed to influence performance during training ${ }^{3}$.

3 One participant with an unusually high error rate at the end of the second block can be observed in the Incongruent training condition. This participant also has unusually slow RT during training. Removal of this participant from this, and all subsequent analyses, has no impact on the pattern or direction of the effects, with the exception of reduced power in most tests - here, the difference is not 
287 Test. Error Rates differed across the different Match conditions (Match Type: $F(1,40)=8.08$, $288 p=.007,{ }_{\mathrm{p}} \eta^{2}=.17$, Observed Power $=.79$ ), with overall lower error rates for the Mismatch 289 condition than for the Match condition. This effect was larger for participants who were trained 290 with item labels that were congruent with the shape of the individual items, and was absent for 291 participants in the incongruent condition (Match Type x Congruence: $F(1,40)=6.38, p=.016$, $292 \mathrm{p}^{2}=.14$, Observed Power $=.69$ ). This finding suggests that the correct detection of large 293 differences between paired images in the mismatch condition was disrupted by having earlier 294 encountered incongruent labels, suggesting sub-optimal processing of the visual properties of the 295 objects. Rejecting a mismatch would typically be easier in a task of this kind, since low-level 296 visual features (e.g., vertical edges, curves) can provide definitive information about a mismatch 297 drawn from different visual categories. Interestingly, for participants who heard incongruent 298 labels during their training, there was no general difference between match and mismatch trials. 299 Effectively, they were worse than would normally be expected at correctly rejecting mismatches. 300 However, as this effect was not predicted, and was somewhat under-powered, it should be 301 treated with caution, and a larger sample size would be required for future investigations seeking 302 to replicate this effect.

303 Reaction times did not differ significantly for participants in the different training conditions ${ }^{4}$.

notable: $F(1,39)=39.83, p<.001, \mathrm{p}^{2}=.51$, Observed Power $=1.00$.

4 In Figure 2B, this can be observed as the outcome of approximately two thirds of participants showing slightly faster responses for ID match than for mismatch (Congruent: 14, Incongruent, 13), overlapping with the remainder of participants, who showed faster responses for mismatch than for ID match (Congruent: 7, Incongruent: 8), but exhibited a larger reaction time difference between trial types. The degree of individual sensitivity to sound symbolism has previously been shown to correlate with extent of sound symbolic congruence in learning (Lockwood \& Dingemanse, 2015). Here, since the direction of this split is the same for both training conditions, it does not appear to arise from sound symbolism, but may index a difference in visual object processing. 

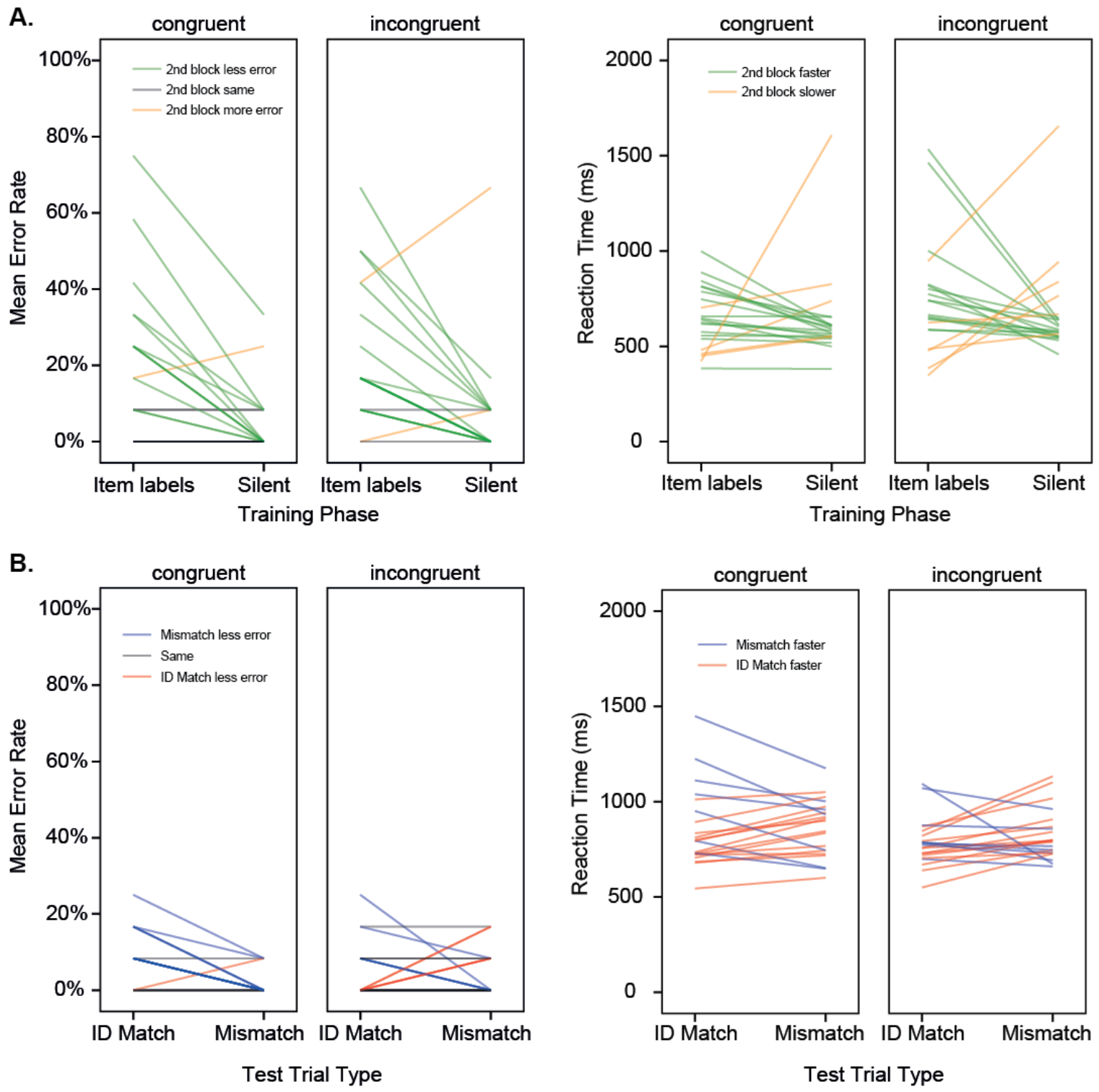

305

306

307

308

309

310

311

312

Figure 2. Experiment 1. Training with item labels. A. Results for the two training phases shown for individual participants in different training conditions. Mean error rate, and mean reaction time (back-transformed from iRT) are shown, where GREEN indicates better performance in the second block (more accurate, faster). C\&D. Results for the two test trial types shown for individual participants in different training conditions. B. Mean error rate, and mean reaction time (back-transformed from iRT) are shown, where BLUE indicates better performance for the Mismatch condition (more accurate, faster). 
317 Table 1. Mean Error rates and Reaction Times (back-transformed from iRT) for Experiment 1.

\begin{tabular}{|c|c|c|c|c|c|c|c|c|c|}
\hline & & \multicolumn{4}{|c|}{ Training } & \multicolumn{4}{|c|}{ Test } \\
\hline & & \multicolumn{2}{|c|}{ Phase 1} & \multicolumn{2}{|c|}{ Phase 2} & \multicolumn{2}{|c|}{ ID Match } & \multicolumn{2}{|c|}{ Mismatch } \\
\hline & & $M$ & $S D$ & $M$ & $S D$ & $M$ & $S D$ & $M$ & $S D$ \\
\hline \multirow[t]{2}{*}{ Error Rate } & Congruent & .19 & .20 & .05 & .09 & .08 & .07 & .02 & .03 \\
\hline & Incongruent & .22 & .18 & .07 & .15 & .05 & .07 & .05 & .06 \\
\hline \multirow[t]{2}{*}{ RT (ms) } & Congruent & 650 & 167 & 637 & 239 & 856 & 212 & 861 & 151 \\
\hline & Incongruent & 750 & 299 & 671 & 251 & 784 & 125 & 825 & 132 \\
\hline
\end{tabular}

319 Experiment 2: Category Labels

320 Each Vinčasso was presented with one of two auditory labels, each of which labeled a category

321 of six visually coherent Vinčasso creatures during the first training block. Half of the participants

322 heard congruent, and half, incongruent item labels. Labels were yoked to categories for all 323 participants in a given condition.

324 Training. Figure 3 shows the results from the training and the test phase of Experiment 2, with 325 the means for individual participants shown separately. Means are given in Table 2. As in 326 Experiment 1, participants solved the categorization task, with a reduction in errors from the first 327 to the second block (Training Phase: $F(1,38)=9.49, p=.004,{ }_{\mathrm{p}} \eta^{2}=.20$, Observed Power $=$ 328 .85). However, unlike in Experiment 1, participants exposed to labels correlated with the 329 category structure were, on the whole, slower to make their category judgments in the second 330 training block, where category labels were not presented $\left(F(1,38)=7.70, p=.009,{ }_{\mathrm{p}} \eta^{2}=.17\right.$, 331 Observed Power $=.72$ ). This effect was not predicted, but suggests that in the first block, 332 participants may have been relying on the diagnostic properties of the audio label to make their 333 category guesses, and subsequently found judgments harder in the silent second block when this 334 label was absent. As elsewhere, no influence of sound symbolism was observed on error rates or 335 reaction times during training. As this effect is somewhat under-powered, they should be treated 336 with caution, and a larger sample size would be required for future investigations seeking to 337 replicate this effect. 
338 Test. As in Experiment 1, participants made more errors accepting an identical pair than they

339 did rejecting a mismatch (Match Type: $F(1,38)=14.00, p=.001,{ }_{\mathrm{p}} \eta^{2}=.27$, Observed

340 Power $=.95$ ). Unlike in Experiment 1, Reaction Time was influenced by a combination of Match

341 Type and Congruence (Match x Congruence: $F(1,38)=21.89, p<.001,{ }_{\mathrm{p}} \eta^{2}=.37$, Observed

342 Power $=.99)$, such that the majority $(17$ of the 21$)$ of participants trained with congruent labels

343 were faster to accept matches than to reject mismatches, while the majority (17 of the 19) of

344 participants trained with incongruent labels were faster to reject mismatches than to accept

345 matches. This finding suggests that passive exposure to labels during training generates

346 differences in object processing, which are in line with the idea that congruent labels facilitate

347 recognition of salient feature similarities, while incongruent labels highlight featural differences.

348 There was also a general difference between the RTs of people in the different training

349 conditions $\left(F(1,37)=4.65, p=.035,{ }_{\mathrm{p}} \eta^{2}=.11\right.$, Observed Power $\left.=.56\right)$, with people in the

350 incongruent condition slightly faster overall during testing. The low power of this group

351 difference suggests it may be artefactual, and a larger sample size would be required for 352 replication. 

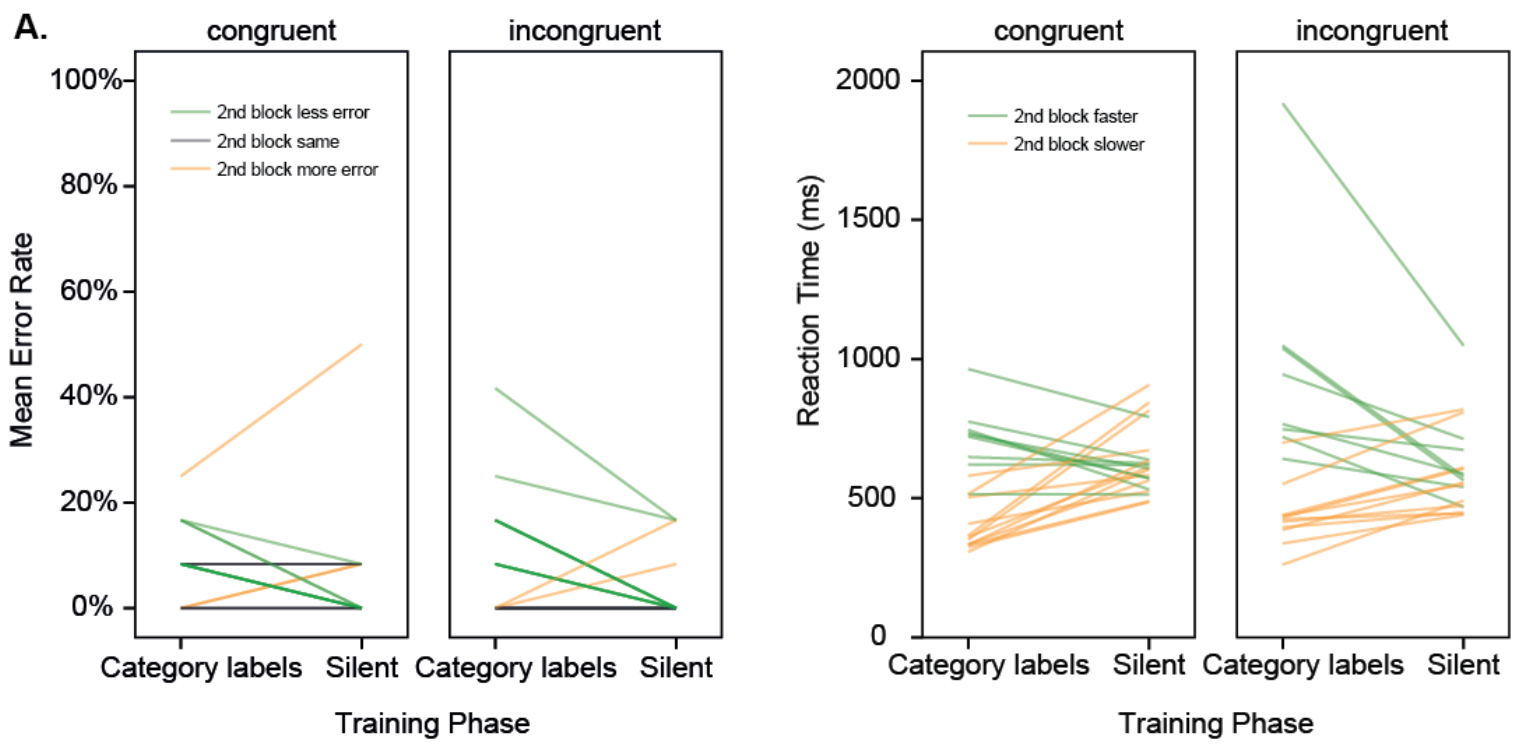

B.
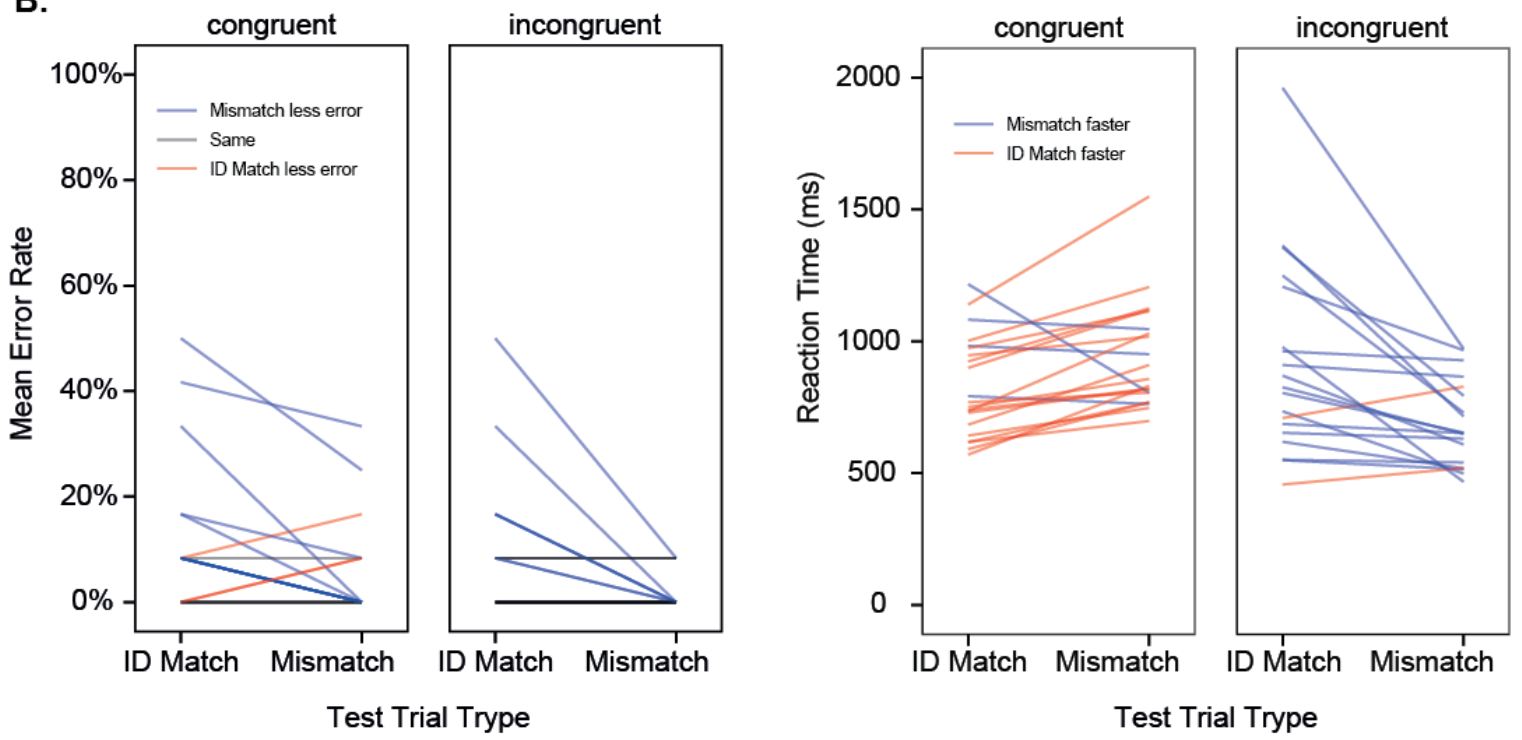

Figure 3. Experiment 2. Training with category labels. A. Results for the two training phases shown for individual participants in different training conditions. Mean error rate, and Mean reaction time (back-transformed from iRT) are shown, where GREEN indicates better performance in the second block (more accurate, faster). B. Results for the two test trial types shown for individual participants in different training conditions. Mean error rate, and Mean reaction time (back-transformed from iRT) are shown, where BLUE indicates better performance for the Mismatch condition (more accurate, faster). 
364 Table 2. Mean Error rates and Reaction Times (back-transformed from iRT) for Experiment 2.

\begin{tabular}{|c|c|c|c|c|c|c|c|c|c|}
\hline & \multicolumn{4}{|c|}{ Training } & \multicolumn{4}{|c|}{ Test } \\
\hline & & \multicolumn{2}{|c|}{ Training 1} & \multicolumn{2}{|c|}{ Training 2} & \multicolumn{2}{|c|}{ ID Match } & \multicolumn{2}{|c|}{ Mismatch } \\
\hline & & $M$ & $S D$ & $M$ & $S D$ & $M$ & $S D$ & $M$ & $S D$ \\
\hline \multirow[t]{2}{*}{ Error Rates } & Congruent & .08 & .07 & .05 & .11 & .11 & .14 & .05 & .09 \\
\hline & Incongruent & .10 & .11 & .03 & .06 & .09 & .13 & .02 & .03 \\
\hline \multirow[t]{2}{*}{ RT (ms) } & Congruent & 534 & 193 & 631 & 117 & 829 & 190 & 940 & 204 \\
\hline & Incongruent & 663 & 385 & 601 & 156 & 918 & 371 & 687 & 165 \\
\hline
\end{tabular}

366 Discussion

367 The key finding of the present research is that the sound symbolism in labels with 368 different ranges of reference (category-label vs item-label) has different outcomes on object 369 processing. Passive exposure to category or item labels during category learning influences later

370 abilities to detect similarities and differences between pairs of visual stimuli: as predicted, 371 category labels influenced the speed of recognizing identical pairs if trained with congruent 372 labels, and the speed of rejecting visually dissimilar pairs, when trained with incongruent labels.

373 In addition, labels disrupted the ability to correctly reject a gross visual difference between two 374 images if the items were trained with incongruent labels. These effects are best understood as 375 arising from differences in visual object encoding grounded in the sound symbolism encountered 376 during the initial training stage. Sound symbolism therefore modulates visual object attention 377 differently when labels align with task-relevant category structure.

378 Until now, the majority of previous studies into sound symbolism have focused on the 379 learnability, guessability or recall of the mapping between a label and its referent. For example, 380 Kovic and colleagues (2010) demonstrated sound symbolic effects in a test phase where 381 participants heard an auditory label, then saw a matching or mismatching picture; Monaghan and 382 colleagues' (2011) participants heard an auditory label and guessed from an array of 12 pictures; 383 and Imai and colleagues (2008) tested participants on extension of action descriptions to novel 384 agents in a two-alternative forced choice task. In all of these studies, the range of reference of the 385 label was unclear, as there was no experimental control of how labels aligned with other 386 information about category structure.

387 By contrast, in the current study, we wanted to know whether sound symbolic relations 
388 encountered during training would change the way that visual objects were encoded, leading to 389 downstream differences in visual object processing, even in the absence of the label. We found 390 that sound symbolism between a label and a category of objects generated predicted differences 391 in visual processing, but that sound symbolism between individual items and their labels did not 392 generate the enhancements that might typically be predicted, in this categorization paradigm. 393 That is to say, sound symbolism affects the formation of category attributes, not item attributes, 394 and those attributes influence later visual object processing. To the best of our knowledge, this is 395 the first study to show that sound symbolism influences visual object processing outside of the 396 mapping between label and referent.

Participants in both experiments were clearly able to infer the category structure from the trials presented in the first training block, as their accuracy improved in the second (silent) training block, where errors fell to below $10 \%$ for participants in all training conditions. In previous research (Kovic, et al., 2010), sound symbolism did not influence the trajectory of category learning in a learning-by-guessing categorization task, with task irrelevant category labels. In the present research, we replicated and extended this observation, with no difference in category learning for congruent versus incongruent labels, when trained with either item or 404 category labels (Experiment 1 and 2, respectively).

Even though the labels presented during the first training block were irrelevant to the task 406 presented in the subsequent test blocks, sound symbolism had pervasive influences on performance in the silent, visual discrimination task: Firstly when trained on categories where each item had different label, participants were worse at rejecting differences than would be typically expected in a task of this kind if they have been trained with congruent labels. This suggests that the normal pattern of object processing may have been disrupted by exposure to 411 incongruent labels. This effect was not predicted, and was somewhat underpowered, so should be 412 treated with some degree of caution. However, if it replicates in future studies, it provides an 413 interesting counterpoint to the perspective that arbitrariness enhances object individuation - it 414 may be the case that incongruent sound-symbolism is qualitatively different to other kinds of 415 artbitrariness.

416 By contrast, when trained on items in labelled categories, participants were better at 417 detecting a match when the labels were congruent, and better at detecting a mismatch when the 
418 labels were incongruent (as shown by their RTs). Hence, sound symbolism in category labels 419 enhances similarity judgments when congruent, and enhances object discrimination when 420 incongruent. These results are consistent with the idea that congruent sound symbolism may 421 enhance the processing of shared object features, in a way that enhances similarity judgments, 422 via efficient visual retrieval processes. We also saw incongruent category labels enhance the 423 discrimination of grossly mismatching picture pairs, which may have been due to enhanced 424 recognition of item differences. We therefore see discrete effects of congruent and incongruent 425 sound symbolism at different levels of labelling.

426 The preceding eighty-odd years of research into linguistic sound symbolism has 427 confirmed that certain sounds in language simply 'go better' with certain perceptual experiences, 428 either via general cross-modal correspondences (Spence, 2011), or communication oriented 429 mechanisms of iconicity (Perniss, Thompson, \& Vigliocco, 2010). These effects are known to be 430 automatic (Parise \& Spence, 2012), unconscious (Hung, Styles, \& Hsieh, 2017), and to enhance 431 memory for individual word meanings under certain conditions (Imai, Haryu, \& Okada, 2005; 432 Kantartzis, et al., 2011; Lockwood, et al., 2016). The finding of differential effects at different 433 level of labelling draws an interesting parallel with existing research into category learning in the 434 absence of sound symbolism.

Previous experimental and modelling work on massed word learning by Monaghan et al. 436 (2011), has shown that both human learners and neural networks will learn to map word-forms to meaning classes faster if the mappings are systematically linked to the meanings, while learning 438 to individuate specific form-meaning pairs is more effective if the mappings are arbitrary. In 439 their studies, the systematicity arises from sound-alike mean-alike relationships analogous to morphology in linguistic systems like grammatical gender (e.g., prefix $\mathrm{X}$ always occurs with 441 word type A). Hence, their kind of systematicity is effective at functionally grouping items 442 which share a similar form, while arbitrariness is better at distinguishing the meanings of 443 individual words. By contrast, linguistic sound symbolism (arising as does from iconic matches 444 between the senses), generally functions at the level of individual pairings between sounds and 445 meanings, rather than at the level of grammatical categories or word classes. Hence, where

446 symbolic congruence gives rise to sound-alike mean-alike relations (due to shared sound447 meaning matching processes), it may be responsible for a type of systematicity, as the 
448 phonological space available for words with similar meanings becomes crowded by an 449 overabundance of similar-sounding words. Gasser (2004), demonstrated that it is possible to 450 efficiently encode a relatively small number of sound symbolic words. The modelling and 451 experimental work of Monaghan, Shillcock, Christinasen and Kirby (2014) has shown a higher 452 proportion of sound-alike mean-alike relations in the early lexicon than the adult lexicon, 453 suggesting that sound symbolism is most useful when the lexicon is still small. This modelling 454 perspective sits well with findings that sound symbolism can facilitate early word learning (Imai, 455 et al., 2008), and may act as a 'Bootstrap' for the acquisition of language (Imai \& Kita, 2014). 456 However, as vocabulary increases, confusability between similar sounding items also increases, 457 as the phonological space becomes crowded, therefore necessitating the emergence of more458 arbitrary linguistic structures.

In the current study, our findings therefore support a dominant theoretical perspective in 460 the field of category labelling: that sound symbolism (like other forms of systematicity) does 461 indeed have its greatest impact at the level of Category labels, rather than Item labels (at least in 462 this task, where category structure is task-relevant), but we also show that the effects of sound 463 symbolism go beyond the 'priming' effects which may be generated by a label, or the strength of 464 the link between the label and referent, as they also influence downstream visual object 465 processing, in the absence of the label.

It should be noted that the two testing conditions were not of the same kind: in the Match 467 condition, the decision can be made purely on visual grounds, and the category of the object cannot form part of the decision process. Whereas in the Mismatch condition, either the learned category difference or the visible item differences could be cues to correctly identifying the mismatch. This discrepancy arises because of an unexpected ambiguity in the wording of out test question, which rendered a third experimental condition un-analysable (For further details, see

472 Supplementary Materials S3). This means at this stage we are not in a position to diagnose 473 whether the effects arise out of purely visual processing, or a combination of visual and category cues. Further studies will be needed to clarify this point, along with the remaining question of whether non-sound-symbolic but otherwise systematic labels generate similar effects. 
Acknowledgements. Thanks go to Ivana Todorović for creating the original Vinčasso

479

480

481

482

483

484

485

486

487

488

489

490

491

492

493

494

495

496

497

498

499

500

501

502

503

504

505

506

507

508

509

510

511

512

513

514

drawings, according to the design parameters, and to Anđela Šoškić for assisting in the collection

of stimulus rating and cloud guessing data. The Cloud Appreciation Society deserves thanks for the final author's sub-ordinate category knowledge of clouds. Preliminary results from some conditions of this project were presented in the poster 'Ković, V., Sučević, J. \& Styles, S.J. "What's in a name?" Category label vs identity label in learning novel categories.' Dubrovnik Conference on Cognitive Science: Language and Conceptual Development, Dubrovnik, Croatia, May 2014.

\section{References:}

Althaus, N., \& Mareschal, D. (2014). Labels direct infants' attention to commonalities during novel category learning. PloS one, 9.(7), e99670.

Althaus, N., \& Westermann, G. (2016). Labels constructively shape object categories in 10month-old infants. Journal of experimental child psychology, 151, 5-17.

Berlin, B. (1995). Evidence for pervasive synesthetic sound symbolism in ethnozoological nomenclature. In L. Hinton, J. Nichols \& J. J. Ohala (Eds.), Sound Symbolism (pp. 7693): Cambridge University Press.

Dingemanse, M., Blasi, D. E., Lupyan, G., Christiansen, M. H., \& Monaghan, P. (2015). Arbitrariness, Iconicity, and Systematicity in Language. Trends in Cognitive Sciences, 19(10), 603-615.

Dingemanse, M., Schuerman, W., Reinisch, E., Tufvesson, S., \& Mitterer, H. (2016). What sound sybolism can and cannot do: Testing the iconicity of ideophones from five langauges. Language, 92(2), e117-e133.

Drijvers, L., Zaadnoordijk, L., \& Dingemanse, M. (2015). Sound-Symbolism is Disrupted in Dyslexia: Implications for the Role of Cross-Modal Abstraction Processes. Paper presented at the 37th Annual Meeting of the Cognitive Science Society.

Edmiston, P., \& Lupyan, G. (2015). What makes words special? Words as unmotivated cues. Cognition, 143, 93-100.

Faul, F., Erdfelder, E., Buchner, A., \& Lang, A.-G. (2009). Statistical power analyses using G*Power 3.1: Tests for correlation and regression analyses. Behavior Research Methods, 41, 1149-1160.

Flumini, A., Ranzini, M., \& Borghi, A. M. (2014). Nomina sunt consequentia rerum-Soundshape correspondences with every-day objects figures. J Mem Lang, 76, 47-60.

Gasser, M. (2004). The origins of arbitrariness of language. Paper presented at the 26th Annual Conference of the Cognitive Science Society, .

Hung, S.-M., Styles, S. J., \& Hsieh, P.-J. (2017). Can a word sound like an object looks before 
515

516

517

518

519

520

521

522

523

524

525

526

527

528

529

530

531

532

533

534

535

536

537

538

539

540

541

542

543

544

545

546

547

548

549

550

551

552

553

554

555

556

557

558

559

560

you have seen it? Sound-shape mapping prior to conscious awareness. Psychological Science, 28(3), 263-275.

Imai, M., Haryu, E., \& Okada, H. (2005). Mapping novel nouns and verbs onto dynamic action events: Are verb meanings easier to learn than noun meanings for Japanese children? Child Development, 76(2), 340-355.

Imai, M., \& Kita, S. (2014). The sound symbolism bootstrapping hypothesis for language acquisition and language evolution. Philosophical Transactions of the Royal Society of London. Series B, Biological Sciences, 369(20130298), 1-14.

Imai, M., Kita, S., Nagumo, M., \& Okada, H. (2008). Sound symbolism between a word and an action facilitates early verb learning. Cognition, 109, 54-65.

Kantartzis, K., Imai, M., \& Kita, S. (2011). Japanese sound-symbolism facilitates word learning in English-speaking children. Cognitive Science, 35, 575-586.

Köhler, W. (1929:1947). Gestalt psychology. New York: Liveright Publishing.

Kovic, V., Plunkett, K., \& Westermann, G. (2010). The shape of words in the brain. Cognition, 114, 19-28.

Lockwood, G., \& Dingemanse, M. (2015). Iconicity in the lab: a review of behavioral, developmental, and neuroimaging research into sound-symbolism. Frontiers in Psychology, 6(1246), 1-14.

Lockwood, G., Dingemanse, M., \& Hagoort, P. (2016). Sound-Symbolism Boosts Novel Word Learning. Journal of Experimental Psychology: Learning, Memory \& Cognition, Advance online. doi: http://dx.doi.org/10.1037/xlm0000235

Lupyan, G., Rakinson, D. H., \& McClelland, J. L. (2007). Language is not Just for Talking: Redundant Labels Facilitate Learning of Novel Categories. Psychological Science, 18(12), 1077-1083. doi: doi:10.1111/j.1467-9280.2007.02028.x.

Lupyan, G., \& Thompson-Schill, S. L. (2012). The evocative power of words: Activation of concepts by verbal and nonverbal means. Journal of Experimental Psychology: General, 141(1), 170-186. doi: 10.1037/a0024904

Maurer, D., Pathman, T., \& Mondloch, C. J. (2006). The shape of boubas: sound-shape correspondences in toddlers and adults. Developmental Science, 9(3), 316-322.

Monaghan, P., \& Christiansen, M. H. (2006). Why form-meaning mappings are not entirely arbitrary in language Paper presented at the Proceedings of the 28th Annual Conference of the Cognitive Science Society.

Monaghan, P., Christiansen, M. H., \& Fitneva, S. A. (2011). The Arbitrariness of the sign: Learning advantages from the structure of the vocabulary. Journal of Experimental Psychology: General(140(3)), 325-347.

Monaghan, P., Mattock, K., \& Walker, P. (2012). The Role of Sound Symbolism in Language Learning. Journal of Experimental Psychology: Learning, Memory \& Cognition, 38(5), 152-1164.

Monaghan, P., Shillcock, R. C., Christiansen, M. H., \& Kirby, S. (2014). How arbitrary is language? Philosophical Transactions of the Royal Society of London. Series B, Biological Sciences, 369(20130299).

Nielsen, A. K. S., \& Rendall, D. (2011). The Sound of Round: Evaluating the Sound-Symbolic Role of Consonants in the Classic Takete-Maluma Phenomenon. Canadian Journal of Experimental Psychology, 65(2), 115-124.

Nielsen, A. K. S., \& Rendall, D. (2013). Parsing the Role of Consonants Versus Vowels in the Classic Takete-Maluma Phenomenon. Canadian Journal of Experimental Psychology,

PeerJ reviewing PDF | (2016:03:9460:2:0:NEW 17 May 2017) 
561

562

563

564

565

566

567

568

569

570

571

572

573

574

575

576

577

578

579

580

581

67(2), 153-163.

Nygaard, L. C., Cook, A. E., \& Namy, L. L. (2009). Sound to meaning correspondences facilitate word learning. Cognition, 112, 181-186.

Parise, C. V., \& Spence, C. (2012). Audiovisual crossmodal correspondences and sound symbolism: a study using the implicit association test. Experimental Brain Research, 220.3-4(3-4), 319-333.

Perniss, P., Thompson, R. L., \& Vigliocco, G. (2010). Iconicity as a general property of language: evidence from spoken and signed languages. Frontiers in Psychology, 1(227). doi: $10.3389 /$ fpsyg. 2010.00227

Ramachandran, V. S., \& Hubbard, E. M. (2001). Synaesthesia-A Window Into Perception, Thought and Language. Journal of Consciousness Studies, 8(123-34).

Sapir, E. (1929). A study in phonetic symbolism. Journal of Experimental Psychology, 12, 225239.

Spence, C. (2011). Crossmodal correspondences: A tutorial review. Attention, Perception and Psychophysics, 73, 971-995.

Sučević, J., Savić, A. M., Popović, M. B., Styles, S. J., \& Ković, V. (2015). Balloons and bavoons vs spikes and shikes: ERPs reveal shared neural processes for shape-soundmeaning congruence in words, and shape-sound congruence in pseudowords. Brain \& Language, 145-146, 11-22. doi: http://dx.doi.org/10.1016/j.bandl.2015.03.011 